\title{
Higgs measurements at the FCC-hh
}

\author{
Michele Selvaggi* \\ CERN, CH-1211 Geneva 23, Switzerland \\ E-mail: michele.selvaggi@cern.ch
}

The future circular hadron-hadron collider FCC-hh is expected to produce collisions at the unrivaled center of mass energy of $\sqrt{s}=100 \mathrm{TeV}$ and to deliver an integrated luminosity of $\mathscr{L}=30 \mathrm{ab}^{-1}$. The FCC-hh offers the unique opportunity to measure the Higgs self-coupling with a $5 \%$ precision. Billions of Higgs bosons will be produced at the FCC-hh, opening a wide range of possibilities in the realm of possible precision Higgs measurements. The Top Yukawa and the Higgs self-coupling can be measured respectively to percent level precision. Final states involving Higgs bosons can be studied in highly boosted kinematical regimes where the impact of systematic uncertainties can be reduced, allowing for percent level precision in most decay chanels including rare decays such as $\mathrm{H} \rightarrow \mu^{+} \mu^{-}$and $\mathrm{H} \rightarrow \mathrm{Z} \gamma$. In addition, the unitarisation of the amplitude the longitudinally polarized vector bosons can be directly tested at the FCC-hh.

The 39th International Conference on High Energy Physics (ICHEP2018)

4-11 July, 2018

Seoul, Korea

${ }^{*}$ Speaker. 


\section{Introduction}

The Future Circular Collider (FCC) is an hypothetical and ambitious accelerator complex in the CERN area designed for the after LHC era. The first step would consist in an electron-positron collider (FCC-ee) that would provide a per-mille level measurement of the Higgs coupling to Z bosons [1]. The second step and main drive on the tunnel and infrastructure design would be a 100 TeV hadron circular collider (FCC-hh). Such center of mass energy can be achieved by means of a $100 \mathrm{~km}$ tunnel and $16 \mathrm{~T}$ bending dipole magnets. The FCC-hh would deliver a peak luminosity of $\mathscr{L}=310^{35} \mathrm{~cm}^{-2} \mathrm{~s}^{-1}$ in its ultimate phase which would result in $\mathrm{O}(15) \mathrm{ab}^{-1}$ of integrated luminosity per experiment. The FCC-hh machine would allow for a direct exploration of massive particles up to $40 \mathrm{TeV}$ [2], improving by approximately one order of magnitude the LHC sensitivity for discovering heavy states. In addition, during its lifetime the FCC-hh is expected to produce tens of billions of Higgs bosons allowing for a rich standard model precision program [3]. Most importantly, a $100 \mathrm{TeV}$ machine will be the only machine allowing for a 5\% measurement of the Higgs self-coupling [3]. In this note we will summarise a subset of possible precision measurements of the Higgs sector that can be performed with the FCC-hh. In the studies presented here, Monte Carlo samples have been generated with the MG5_aMC@ NLO 2.5.2 package [4], showered and hadronised with PYTHia 8.230 [5]. The detector simulation was performed with the fast simulation framework DELPHES 3.4.2 [6] assuming the FCC-hh detector performance. A detailed description of the results presented here can be found in [7].

\section{The Higgs self-coupling}

A precise measurement of the Higgs self-coupling $\lambda$ probes the shape of the Higgs potential near our vacuum and could provide insights about the electro-weak phase transition (EWPT). The Higgs self-coupling can be probed via double Higgs production. After the full LHC run at high luminosity (HL-LHC), the SM sensitivity to the self-coupling will be barely reached. In contrast, at $\sqrt{s}=100 \mathrm{TeV}$, the di-Higgs production cross section increases by a factor 50, allowing for a precise measurement of this fundamental SM parameter.

The Higgs self-coupling can be probed via a number of different Higgs boson decay channels. Given the small cross-section, typically at least one of the Higgs bosons is required to decay to a pair of b-quarks. Here, we consider 5 channels: $\mathrm{HH} \rightarrow \mathrm{bb} \gamma \gamma, \mathrm{HH} \rightarrow \mathrm{bb} \tau \tau, \mathrm{HH} \rightarrow \operatorname{bbZZ}(4 \ell)$, $\mathrm{HH} \rightarrow 4 \mathrm{~b}+\mathrm{jet}$, and $\mathrm{HH} \rightarrow \mathrm{bbWW}$. The di-photon invariant mass spectrum of the signal and the single Higgs and QCD backgrounds obtained in the bb $\gamma \gamma$ channel is shown in Figure 1 (left). Figure 1 (right) shows a summary of the achievable precision in the aforementionned decay channels. The ultimate achievable precision on the self-coupling will be obtained via a combination of the sensitivities in the various decay channels and has yet to be performed. The highest precision is achieved in the bb $\gamma \gamma$ channel with $\delta \kappa_{\lambda} \approx 6 \%$. The bb $\tau \tau$ channel has been investigated in Ref. [8] and is expected to provide a $10 \%$ measurement on the Higgs self-coupling. The comparison of the sensitivity that can be achieved in the various decay channels is shown in Figure 1 (right).

\section{Single Higgs measurements}

Measurements of the Higgs couplings to photons or $\mathrm{Z}$ bosons (the latter being performed in the 

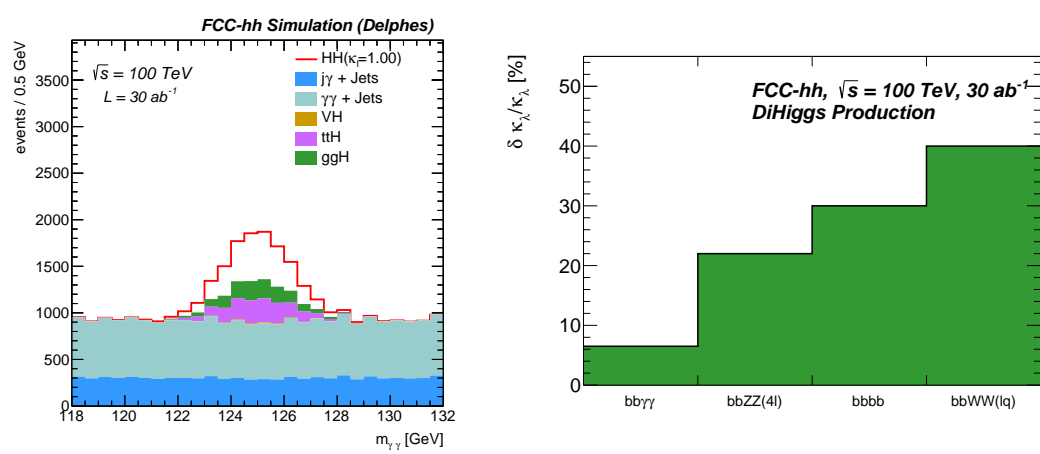

Figure 1: Left: Di-photon candidates invariant mass spectrum after applying all selection criteria. Right: Expected precision on the measurements of the Higgs self-coupling in the various decay channels.

$\mathrm{H} \rightarrow \mathrm{ZZ}^{*} \rightarrow 4 \ell$ channel) will benefit from very large statistics at the HL-LHC and the achievable precision in such channels will be limited by systematics $[9,10]$. In contrast, rare Higgs decays such as $\mathrm{H} \rightarrow \mu^{+} \mu^{-}$or $\mathrm{H} \rightarrow \mathrm{Z} \gamma$ will be statistically limited at the HL-LHC $[9,10]$. At $\sqrt{s}=100 \mathrm{TeV}$, the Higgs production rate increases by a factor 15 for gluon-fusion production $(\mathrm{ggF})$ with respect to $\sqrt{s}=14 \mathrm{TeV}$, and up to a factor 55 for associated production with a $t \bar{t}$ pair (ttH) [3]. With a total integrated luminosity of $\mathscr{L}=30 \mathrm{ab}^{-1}$ at the FCC-hh, we expect a factor $\approx 100$ increase in the total number of produced Higgs events compared to HL-LHC, leading to an overall reduction of a factor $\approx 10$ of statistical uncertainty in Higgs-related measurements.

We focus here on a subset of Higgs decay channels: $\mathrm{H} \rightarrow \mathrm{ZZ}^{*} \rightarrow 4 \ell, \mathrm{H} \rightarrow \gamma \gamma, \mathrm{H} \rightarrow \mu^{+} \mu^{-}$ and $\mathrm{H} \rightarrow \mathrm{Z} \gamma \rightarrow \ell^{+} \ell^{-} \gamma$, where $\ell=\mathrm{e}, \mu$. Abundant statistics can be exploited by explicitly selecting phase space regions where systematic uncertainties are small and better understood. The expected precision in these channels has been studied as a function of the minimal requirement on the Higgs reconstructed transverse momentum which can be fully reconstructed from the visible final state products. Requiring the Higgs at high momentum presents the advantage of high $\mathrm{S} / \mathrm{B}$, as well as lower systematics. The expected $\delta \mu / \mu$ is found to be to $1 \%$ up to $p_{\mathrm{T}}(\mathrm{H})=200 \mathrm{GeV}$ in all channels [7]. In addition one can measure ratios of couplings thus cancelling out theoretical uncertainties on the production cross-section, luminosity uncertainties and uncertainties on the final state object reconstruction efficiencies. Estimates for the precision that can be obtained at the FCC-hh on the ratios $\mathrm{BR}\left(\mathrm{H} \rightarrow \mu^{+} \mu^{-}\right) / \mathrm{BR}(\mathrm{H} \rightarrow 4 \mu)$ and $\mathrm{BR}(\mathrm{H} \rightarrow \gamma \gamma) / \mathrm{BR}(\mathrm{H} \rightarrow 2 e 2 \mu)$ are shown in Figure 2. Such relative measurements are particularly interesting since they can be re-interpreted as absolute measurements, provided that the branching fraction in the denominator (i.e. the $\mathrm{H} \rightarrow \mathrm{ZZ}$ coupling) has been measured previously at high precision.

\section{Conclusion and outlook}

The FCC-hh $100 \mathrm{TeV}$ pp collider will present a unique opportunity for measuring key standard model parameters such as the Higgs self-coupling and Higgs rare decay branching ratios. In this note we have restricted our discussion to a subset of possible measurement of the Higgs sector at $\sqrt{s}=100 \mathrm{TeV}$. We have shown that percent level precision is achievable in rare decay channels such as $\mathrm{H} \rightarrow \mu^{+} \mu^{-}$and $\mathrm{H} \rightarrow \mathrm{Z} \gamma$ as well as on ratios of Higgs couplings. We have also shown that 

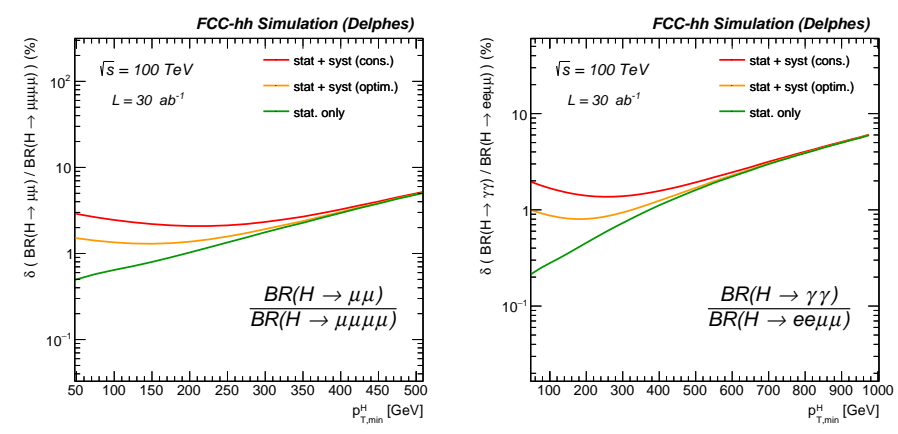

Figure 2: Expected precision on the ratio $\mathrm{BR}\left(\mathrm{H} \rightarrow \mu^{+} \mu^{-}\right) / \mathrm{BR}(\mathrm{H} \rightarrow 4 \mu)$ (left) and $\mathrm{BR}(\mathrm{H} \rightarrow \gamma \gamma) / \mathrm{BR}(\mathrm{H} \rightarrow 2 e 2 \mu)$ (right) as a function of the minimal requirement on the Higgs reconstructed transverse momentum. The expected precision is given for three scenarios: only statistical uncertainties are included (stat-only), statistical and optimistic (conservative) systematics on the object reconstruction efficiencies.

$5 \%$ precision on the Higgs self-coupling can be achieved with $\mathscr{L}=30 \mathrm{ab}^{-1}$. Several additional measurements can be performed in the Higgs sector at the FCC-hh. Percent level accuracy on the top Yukawa can be obtained from the measurement of the ratio of ttH and $\mathrm{ttZ}$ cross-sections [11]. In addition, a measurement of the rate of longitudinally polarized vector bosons can allow for the first time to directly test the unitarisation of the amplitude of $W_{L} W_{L}$ scattering at very high energies.

\section{References}

[1] D. d'Enterria, Physics at the FCC-ee, in proceeding of 17th Lomonosov Conference on Elementary Particle Physics 2017 [hep-ex/1602.05043].

[2] T. Golling et al., Physics at a 100 TeV pp collider: beyond the Standard Model phenomena, CERN Yellow Report 3 441-634 (2017) [hep-ph/1606.00947].

[3] R. Contino et al., Physics at a $100 \mathrm{TeV}$ pp collider: Higgs and EW symmetry breaking studies, CERN Yellow Report 3 255-440 (2017) [hep-ph/1606.09408].

[4] J. Alwall et al., MadGraph 5 : Going Beyond, JHEP 06128 (2011) [hep-ph/1106. 0522].

[5] T. SjÃústrand et al., An Introduction to PYTHIA 8.2, Comput. Phys. Commun. 191 159-177 (2015) [hep-ph/1410.3012].

[6] J. de Favereau et al., DELPHES 3, A modular framework for fast simulation of a generic collider experiment, JHEP 02057 (2014) [hep-ex/1307. 6346].

[7] M. Selvaggi, Higgs measurements at FCC-hh, CERN Notes [https://cds.cern.ch/record/2642471].

[8] S. Banerjee et al., hh+jet production at 100 TeV, Eur. Phys. J. C78 322 (2018) [hep-ph/1802.01607].

[9] CMS Collaboration, Projected performance of Higgs analyses at the HL-LHC for ECFA 2016, CMS Report CMS-PAS-FTR-16-002 (2016).

[10] ATLAS Collaboration, Projections for measurements of Higgs boson signal strengths and coupling parameters with the ATLAS detector at a HL-LHC, ATLAS Report ATL-PHYS-PUB-2014-016 (2016).

[11] M.L. Mangano et al., Measuring the Top Yukawa Coupling at 100 TeV, Eur. Phys. J. G43 035001 (2016) [hep-ph/1507.08169]. 